Research paper

Clinical microbiology

\title{
Bacteroides fragilis: A whole MALDI-based workflow from identification to confirmation of carbapenemase production for routine laboratories
}

\author{
M. Cordovana ${ }^{\text {a, * }}$, M. Kostrzewa ${ }^{\text {b }}$, J. Sóki ${ }^{\text {c }}$, E. Witt ${ }^{\text {d }}$, S. Ambretti ${ }^{\text {a }}$, A.B. Pranada ${ }^{\text {d }}$ \\ a Microbiology Unit of the University Hospital, Policlinico Sant'Orsola-Malpighi, Bologna, Italy \\ ${ }^{\mathrm{b}}$ Bruker Daltonik GmbH, Bremen, Germany \\ c Institute of Clinical Microbiology, University of Szeged, Szeged, Hungary \\ d Department of Medical Microbiology, MVZ Dr. Eberhard \& Partner Dortmund, Dortmund, Germany
}

\section{A R T I C L E I N F O}

\section{Article history:}

Received 1 February 2018

Received in revised form

30 March 2018

Accepted 3 April 2018

Available online $\mathrm{xxx}$

\begin{abstract}
A B S T R A C T
Bacteroides fragilis is a frequent anaerobic pathogen and can cause severe infections. Resistance to carbapenems, associated with the cfiA gene encoded carbapenemase, represents an emerging problem. To date, no rapid methods are available to detect and confirm this resistance mechanism in routine laboratories, and the missed recognition of carbapenemase-producing strains can lead to therapeutic failures. In this study we have investigated a whole MALDI-TOF MS-based workflow to detect carbapenemaseproducing $B$. fragilis, using the largest set of $B$. fragilis clinical isolates ever tested. The presence of the cfiA gene was predicted by MALDI subtyping into Division I (cfiA-negative) or Division II (cfiA-positive). The carbapenemase activity in cfiA-positive strains was confirmed by a MALDI-TOF MS imipenem hydrolysis assay (MBT STAR-Carba, Bruker Daltonik, Germany), that was further used for a characterization of the strains in terms of cfiA expression level.

The validity of MALDI subtyping was verified by PCR for the cfiA gene, while results of MALDI hydrolysis assay were compared to conventional methods for susceptibility testing and carbapenemase detection (Carba-NP and disk diffusion synergy test). A genetic analysis of the IS elements upstream cfiA was performed, for the evaluations regarding the expression level of cfiA. A total of $5300 \mathrm{~B}$. fragilis isolates (406 from Bologna, Italy, and 4894 from Dortmund, Germany) were identified and subtyped by MALDI-TOF MS, yielding 41/406 (10.1\%) strains from Bologna and 374/4894 (7.6\%) from Dortmund to belong to Division II. Molecular verification by PCR for the cfiA gene on a subset of strains confirmed the MALDI typing results in all cases (sensitivity and specificity of 100\%). MBT STAR-Carba assay detected the carbapenemase activity in all of the 70 cfiA-carrying strains tested. Moreover, it allowed distinct separation into slow (59) and fast (11) imipenem hydrolyzers corresponding to cfiA expression levels as well as to low or high MICs for carbapenems, respectively. Among the 11 cfiA-positive strains with high carbapenem MIC, only 7 harboured IS elements upstream the carbapenemase gene showing low expression level as well.

The MALDI-TOF MS-based workflow was superior to the currently available phenotypic methods for carbapenemase detection as it proved to be more sensitive and accurate than Carba NP and disk diffusion synergy test. The whole MALDI-TOF MS-based workflow allows an accurate identification of $B$. fragilis clinical strains with reliable classification into Division I/II, and confirmation of the carbapenemaseproduction, together with estimation of carbapenemase activity, within less than $2 \mathrm{~h}$. This may be of particular interest for early therapeutical decisions in life-threatening infections.
\end{abstract}

(c) 2018 Elsevier Ltd. All rights reserved.

\footnotetext{
* Corresponding author. via della salita, 21, 40178, Bologna, Italy.

E-mail address: miri-78@live.it (M. Cordovana).
}

\section{Introduction}

Bacteroides fragilis is an opportunistic pathogen and may cause 
severe and life-threatening endogenous infections. Although counting for only $0.5 \%$ of the human colonic flora, $B$. fragilis is the most important Gram-negative anaerobic pathogen implicated in almost every type of infection [1,2]. Furthermore $B$. fragilis strains are the most antibiotic resistant organisms among anaerobes [3], and their resistance rates have increased in the past two decades [4-6], with a growing number of multidrug resistant (MDR) isolates reported [7-9]. Carbapenems are one of the most effective treatment options for monobacterial or mixed infections involving $B$. fragilis, but carbapenem resistance is an emerging problem among these strains. A steady trend toward higher MICs of carbapenems has been observed among $B$. fragilis strains since the 2000s, presumably due to increased antibiotic pressure [10-12].

Resistance to carbapenems in $B$. fragilis is mediated by the cfiA gene (also known as ccrA), located on the chromosome (even if exceptions were described) [13], and restricted to Division II of the species [14]. CfiA encodes a very powerful periplasmatic Ambler class B di-zinc metallo-beta-lactamase, so far, the unique carbapenemase enzyme found in $B$. fragilis, that confers resistance to all beta-lactam agents, including beta-lactam/beta-lactamase inhibitor combinations $[15,16]$. CfiA can be either silent or expressed at different levels, resulting in a broad spectrum of MIC values of carbapenems, ranging from susceptible to resistant phenotype [17]. The expression level was shown to be mainly related to the presence of a variety of IS elements carrying outward-oriented promoters, that enhance the carbapenemase expression when inserted upstream of the cfiA sequence [18], although resistant strains without such IS elements have also been identified [14,19]. The spread of cfiA is underestimated, because only strains with the activated genes and high MIC values can be detected by routine susceptibility testing, or surveillances. Nevertheless, under selective pressure during therapy, cfiA positive strains with low MIC values can undergo a genetic re-arrangement, that results in the expression of full resistance to carbapenems, leading to therapeutic failure $[20,21]$.

Routine practice for identification and determination of antimicrobial susceptibility in anaerobes varies between countries and laboratories. Most laboratories usually reserve such analyses for cases where an anaerobic isolate is the predominant pathogen, since traditional methods for identification and susceptibility testing are time-consuming, require expertise, and present problems of interpretation, reproducibility and standardization [22-24]. To date, no routine tools are available to detect cfiA-harbouring strains and to confirm their carbapenemase production. Conventional methods to detect carbapenem-resistance are not intended for use with anaerobes, moreover they are slow (24-48 h), and may lack sensitivity for detection of low-level resistance $[25,26]$. Genotypic methods, such as PCR or WGS are not suitable for routine application [27].

The introduction of matrix assisted laser desorption ionization time-of-flight mass spectrometry (MALDI-TOF MS) greatly improved routine identification at species level of anaerobes, providing a quality of results comparable with molecular methods [28-30]. Moreover, recently, it has also been proven that MALDITOF MS method is able to differentiate Division I and II of $B$. fragilis directly through characteristics in mass spectra, thereby cfiAharboring strains can be detected during the identification process, directly [31,32]. Furthermore, the possibility to verify the carbapenemase activity by detecting the cleavage of the antibiotic molecule after the incubation with the bacterial strain tested has been described $[33,34]$. However, the possibility to characterize the cfiA-harbouring strains in terms of expression level of the enzyme by MALDI-TOF MS application has not been demonstrated yet.

The aim of this study was to investigate the applicability in routine of a whole MALDI-TOF MS-based diagnostic approach for the detection of carbapenem-resistance in B. fragilis, analyzing two large collections of clinical isolates from an Italian and a German laboratory. The workflow comprises the identification of $B$. fragilis strains, the differentiation of the isolates in Division I and II, and the confirmation of the carbapenemase activity by an imipenem hydrolysis assay by MALDI-TOF MS. Furthermore, we compared the applicability of classical phenotypic methods and the MS-based method for detection of the carbapenemase activity in case of a specially selected group of cfiA-positive $B$. fragilis strains.

\section{Materials and methods}

\subsection{Strains included to the study}

Altogether, 5300 non-duplicated $B$. fragilis strains were isolated in two routine clinical microbiology laboratories. From March 2013 to May 2017406 B. fragilis strains were isolated in the Microbiology Unit of the University Hospital (Bologna, Italy) and 4894 strains were isolated from November 2009 to May 2017 in the Department of Medical Microbiology of the MVZ Dr. Eberhard \& Partner (Dortmund, Germany). The strains were cultured on Tryptose Soy Agar with 5\% sheep blood (Meus, Piove di Sacco, Italy) in Bologna, and on Schaedler Agar with $5 \%$ sheep blood (BD, Heidelberg, Germany) in Dortmund, for $24-72 \mathrm{~h}$ at $35^{\circ} \mathrm{C}$, in anaerobic environment, achieved with AnaeroGen jars (Oxoid, Basingstoke, UK) or Anoxomat jar system (MART Microbiology, Drachten, The Netherlands). Identification of the isolates at species level was performed in the collecting laboratories, using the MALDI Biotyper 3.1 system (Bruker Daltonik, Germany), according to the manufacturer's instructions. High confidence species identification was accepted, if the $\log$ (score) was $\geq 2.00$, low confidence species identification $\log$ (score) values $(\geq 1.70$ and $<2.00$ ) were accepted if the three best matches showed the same species name. Any results with $\log$ (score) $<1.70$ were considered as an unacceptable identification. Strains were kept at $-80^{\circ} \mathrm{C}$ for further testing.

Beyond susceptibility testing performed during routine practice in the collecting laboratories, a selection of $n=143$ strains was chosen for further investigations. This subset of isolates included all the Division II strains isolated from 2014 onwards ( $n=70,29$ from Bologna, 41 from Dortmund), and a similar number of Division I strains, randomly chosen as negative controls $(n=73)$. All these strains underwent susceptibility testing for carbapenems in Bologna using standardized conditions. Etest methodology (M.I.C. Evaluator, Oxoid, UK) was applied according to the EUCAST guidelines, version 6.0 (http://www.eucast.org/ast_of_bacteria/ previous_versions_of_documents/). Meropenem MICs were measured for all these selected strains, while imipenem MICs were determined for those, which were proven to belong to Division II and harboured the cfiA gene.

Typing of B. fragilis strains by MALDI-TOF MS, confirmation of the presence of the cfiA gene by PCR and investigation of the IS elements.

All the $B$. fragilis strains with accepted identification on species level were further investigated to differentiate those belonging to Division II by the MALDI Biotyper system at the collection sites as described earlier [31]. The routine identification spectra were further analyzed by means of MALDI Biotyper 3.1 software by matching against the previously published $c f i A$-positive (Division II $B$. fragilis) and cfiA-negative (Division I B. fragilis) MSP library. The above mentioned subgroup of $n=143$ selected strains (all the $\mathrm{n}=70$ strains which belonged to Division II by MALDI-TOF MS isolated from 2014 onwards, and $n=73$ strains categorized as Division I by MALDI-TOF MS analysis) underwent confirmation for the 
presence of the cfiA gene by PCR. PCR analysis was performed on a PTC-200 thermal cycler (MJ Research, St. Bruno, Canada) using $0.5 \mu \mathrm{M}$ of the primers cfiA1 and cfiA2 described in Sóki et al. [35] and $12.5 \mu \mathrm{l}$ of a $2 \times$ ABsoluteBlue QPCR Mix (ThermoFisher Scientific, Waltham, USA). Reaction volume was adjusted to $25 \mu \mathrm{l}$ with PCR grade water. The cycling parameters were as follows: $95{ }^{\circ} \mathrm{C}$ $15 \mathrm{~min} ; 94^{\circ} \mathrm{C} 30 \mathrm{~s}, 62{ }^{\circ} \mathrm{C} 30 \mathrm{~s}, 72^{\circ} \mathrm{C} 60 \mathrm{~s}, 30 \times ; 72^{\circ} \mathrm{C}$ for $5 \mathrm{~min}$.

The integration of IS elements into upstream regions of the cfiA genes was determined by end-point PCR for all cfiA-positive strains as described earlier [35].

\subsection{Detection of carbapenemase production and activity of the enzyme by MBT STAR-Carba method}

The selection of $\mathrm{n}=143 B$. fragilis strains chosen for deeper investigations, both $c f i A$-positive and $c f i A$-negative isolates, was investigated by MBT STAR-Carba kit (Bruker Daltonik, Bremen, Germany) to verify the carbapenemase activity. Application of this kit for anaerobes is off-label. The assay was performed according to manufacturer's instructions for aerobic bacteria with slight modifications. In brief, a $1 \mu$ l loop of bacteria was suspended in $50 \mu \mathrm{l}$ imipenem solution included in the kit. The suspensions were incubated at $35^{\circ} \mathrm{C}$ on a shaker for $30 \mathrm{~min}$, centrifuged for $2 \mathrm{~min}$, and $1 \mu \mathrm{l}$ of the supernatant was spotted in duplicate directly onto a polished steel MALDI target plate. The pellet was immediately resuspended again, incubated for further $30 \mathrm{~min}$, centrifuged for $2 \mathrm{~min}$, and a second spotting step was performed from the supernatant, in order to record, for each sample, the imipenem mass spectra after 30 and $60 \mathrm{~min}$ incubation time. The spots were air-dried, overlaid with MBT STARCarba matrix solution. Once dried, the MALDI target plate was introduced into the Microflex LT mass spectrometer for automatic measurement.

For each run, a positive control (PCR-confirmed KPC-producing E. coli) and two negative controls (E. coli ATCC 25922 and $B$. fragilis ATCC 25285) were also tested. Measurements of mass spectra were performed using the MBT Compass STAR-BL method for spectra acquisition. The parameter settings were: ion source 1 $19.00 \mathrm{kV}$, ion source $217.05 \mathrm{kV}$, lens $6.0 \mathrm{kV}$, detector gain $2.5 \mathrm{kV}$, laser frequency $60 \mathrm{~Hz}$. Spectra were recorded in the mass range of 100-1000 Da. For each spot, two spectra were acquired, and each spectrum was obtained from the sum of 240 laser shots. For calibration, the antibiotic calibration standard (ACS - Bruker Daltonik) was used according to instructions of the manufacturer. The analysis of imipenem spectra was performed using a software prototype with the identical parameters used in the actual Bruker MBT STAR-Carba software module. It automatically calculates the logarithmic ratio of the intensity of the internal standard and the intensity of the non-hydrolyzed imipenem molecules, and the logRQ values of the samples and the controls $(\log R Q=\log [$ internal standard peak intensity]/[sum non-hydrolyzed peak intensities]). The $\operatorname{logRQ}$ values of the samples are normalized against the $\operatorname{logRQ}$ values of the negative and positive controls, and displayed as normplots. LogRQ values 0.00 and 1.00 correspond to $0 \%$ and $100 \%$ of hydrolytic activity, respectively. LogRQ values $\leq 0.2$ indicate an absence of hydrolyzing activity, $\log R Q$ values $\geq 0.4$ indicate the presence of a carbapenemase activity. LogRQ values between 0.2 and 0.4 means an ambiguous degree of hydrolysis, that requires a re-testing. For each strain, the comparison between the logRQ results at $30 \mathrm{~min}$ and at $60 \mathrm{~min}$ was performed, to investigate whether a correlation between the rate of hydrolysis and the carbapenem MIC values could be demonstrated, and thereby a characterization of the strains in terms of expression level of cfiA was possible.

\subsection{Detection of carbapenemase production by classical methods (Carba NP and disc-diffusion synergy test)}

A subset of $29 B$. fragilis strains belonging to Division II were further tested off-label with the available routine methods used to detect carbapenemase production in Enterobacteriaceae. Namely the Carba NP test (Neo-Rapid CARB-Kit, Rosco Diagnostics, Taastrup, Denmark) and the disk diffusion synergy test (KPC/MBL Confirm Kit - Rosco Diagnostica, Taastrup, Denmark) were evaluated whether they could be a reliable option to detect also the cfiArelated carbapenemase activity in a group of $B$. fragilis strains with different MICs for meropenem (1- $\geq 32 \mathrm{mg} / \mathrm{L})$. Both tests were performed following the manufacturer's instructions. To perform synergy test, the modifications required for the growth of anaerobic bacteria were applied (Brucella blood agar (Meus, Piove di Sacco, Italy) and incubation in anaerobic atmosphere).

\section{Results}

\subsection{Identification of $B$. fragilis isolates and determination of the number of isolates belonging to division II in the two centres}

In Bologna, 406 B. fragilis clinical isolates were identified by MALDI-TOF MS at species level during the test period. Overall ID log score values were between 1.817 and 2.615 (average $=2.398$, median $=2.435, \mathrm{SD}=0.124)$. During a longer testing period in Dortmund 4894 B. fragilis isolates were identified by MALDI-TOF MS at species level, with overall ID log score values between 1.740 and 2.700 (average $=2.411$, median $=2.430, \mathrm{SD}=0.110$ ). The number of $B$. fragilis strains belonging to Division II were 41 of $406(10.1 \%)$ in Bologna and 374 of 4894 (7.6\%) in Dortmund. Altogether 415 of 5300 B. fragilis (7.8\%) recent clinical isolates were found by the MALDI-TOF MS typing method to belong to Division II, suspicious to harbour the cfiA gene in an active or inactive form. When we evaluated the prevalence of Division II isolates in relation to the source of the specimens, we could observe a significant difference between Bologna and Dortmund regarding blood cultures. First, we found that in Bologna, 17 of the 41 (41.5\%) B. fragilis strains, which belonged to Division II, had been isolated from blood cultures, while in Dortmund only 7 of 374 (1.9\%) Division II B. fragilis isolates were obtained from blood cultures. Second, we found that in Bologna, the prevalence of Division II strains resulted higher among isolates from blood cultures (15.2\%), in comparison with strains isolated from all other specimens (8.1\%), whereas it was $6 \%$ and $7.7 \%$, respectively, in Dortmund.

\subsection{Investigation of a subset of $B$. fragilis isolates belonging to division I and II for the presence of cfiA gene and the IS elements}

In all $70 \mathrm{~B}$. fragilis isolates, belonging in Division II according to the MALDI-TOF MS typing results (29 from Bologna and 41 from Dortmund), available for further studies, PCR confirmed the presence of the cfiA gene. In the case of the other 73 selected $B$. fragilis strain which belonged to Division I, none of them gave a positive PCR result for the cfiA gene (Table 1 ). Out of the $70 B$. fragilis strains with positive PCR results for the cfiA gene only 7 harboured IS elements upstream of the carbapenemase gene. All strains had a meropenem MIC $\geq 16 \mathrm{mg} / \mathrm{L}$. A great variety of the IS elements was found among these strains, such as IS613 (in two isolates), IS614B (in two isolates), IS942, IS1169, and IS1187 (each in one isolate) (Table 1). No IS elements were detected upstream of the cfiA gene in the strains with lower meropenem MICs $(<16 \mathrm{mg} / \mathrm{L})$.

MICs of meropenem and imipenem for a subset of B. fragilis isolates belonging to Division I and II.

The $B$. fragilis strains belonging to Division I, being cfiA-negative 
Table 1

Presence of $c$ fiA and IS elements among a subset of $B$. fragilis strains belonging to Division I and II.

\begin{tabular}{lll}
\hline B. fragilis (No of isolates) & \multicolumn{2}{l}{ Number of strains positive for } \\
\cline { 2 - 3 } & cfiA & IS elements \\
\hline Division I (73) & & nt $^{\mathrm{a}}$ \\
Bologna (30) & 0 & $\mathrm{nt}^{\mathrm{a}}$ \\
Dortmund (43) & 0 & \\
Division II (70) & & 3 \\
Bologna (29) & 29 & 3 (IS942, IS613, IS614B) \\
MIC MER $\geq 16$ & 5 & 0 \\
MIC MER $<16$ & 24 & 4 \\
Dortmund (41) & 41 & 4 (IS613, IS614B, IS1169, IS1187) \\
MIC MER $\geq 16$ & 6 & 0 \\
MIC MER $<16$ & 35 & \\
\hline a Nt: not tested. & & \\
b MER meropenem. & &
\end{tabular}

in PCR assay, showed meropenem MIC values between $0.015 \mathrm{mg} / \mathrm{L}$ and $2 \mathrm{mg} / \mathrm{L}$ with slight differences according to the two isolation centres, confirming full susceptibility of the isolates to carbapenems. The cfiA-positive strains all proven to belong to Division II by the MALDI-TOF MS-based typing, showed meropenem MIC values between 0.12 and $\geq 32 \mathrm{mg} / \mathrm{L}$, and the imipenem MIC values were between $0.06 \mathrm{mg} / \mathrm{L}$ and $\geq 32 \mathrm{mg} / \mathrm{L}$. Differences in the distributions of MIC values both for meropenem and imipenem of $c$ fiA-positive strains isolated in Bologna and Dortmund were observed, demonstrating a higher level of resistance in $B$. fragilis strains isolates in Bologna (Table 2).

\subsection{Detection of carbapenemase-production in B. fragilis division II strains by the MBT STAR-Carba assay}

In this assay, we included all those isolates, independent of the meropenem or imipenem MICs measured (Table 2), which gave positive PCR results for the cfiA gene (altogether 70 isolates). As negative control, $33 \mathrm{~B}$. fragilis isolates with negative PCR results for the cfiA gene were randomly selected from the $B$. fragilis strains belonging to Division I. The STAR-Carba assay detected the imipenem hydrolysis in all 70 cfiA-positive $B$. fragilis strains, and in none of the 33 cfiA-negative strains involved in this part of the study independent of the MICs of meropenem. The $11 \mathrm{~B}$. fragilis strains with high meropenem MIC values $(\geq 16 \mathrm{mg} / \mathrm{L}$ ) showed a full imipenem hydrolysis after $30 \mathrm{~min}$, while the 59 strains with lower meropenem MIC value ( $<16 \mathrm{mg} / \mathrm{L}$, ranged between 0.125 and $8 \mathrm{mg} /$ L) achieved it after 60 min (Fig. 1, Table 3).

The logRQ score values at 30 min showed a correlation with the meropenem and imipenem MIC values $(r=0.57$ and $r=0.77$, respectively), regardless the absolute value of the logRQ score.

The comparison between $\log R \mathrm{Q}$ values at $30 \mathrm{~min}$ and at $60 \mathrm{~min}$ $\left(\Delta \log R_{60 \mathrm{~min}-30 \mathrm{~min}}\right)$ showed a minimal increase or a decrease of imipenem hydrolysis (average -0.14) among the 11 strains with

Table 2

Distribution of imipenem and meropenem MICs of selected B. fragilis strains belonging to Division II and I originating from two centres.

\begin{tabular}{|c|c|c|c|c|c|c|}
\hline \multirow[t]{2}{*}{ Strain isolated in (No) } & \multicolumn{3}{|c|}{ Imipenem MIC (mg/L) } & \multicolumn{3}{|c|}{ Meropenem MIC (mg/L) } \\
\hline & MIC range & $\mathrm{MIC}_{50}$ & $\mathrm{MIC}_{90}$ & MIC range & $\mathrm{MIC}_{50}$ & $\mathrm{MIC}_{90}$ \\
\hline \multicolumn{7}{|l|}{ B. fragilis Division II } \\
\hline Bologna (29) & $0.125-\geq 32$ & 4 & $\geq 32$ & $1-\geq 32$ & 4 & $\geq 32$ \\
\hline Dortmund (41) & $0.062-32$ & 1 & 32 & $0.125-\geq 32$ & 2 & $\geq 32$ \\
\hline \multicolumn{7}{|l|}{ B. fragilis Division I } \\
\hline Bologna (30) & - & - & - & $0.015-2$ & 0.125 & 0.5 \\
\hline Dortmund (43) & - & - & - & $0.015-1$ & 0.062 & 0.25 \\
\hline
\end{tabular}

high carbapenem MICs, while this increase was significant among the strains with lower carbapenem MICs (average + 0.58) (Table 3).

Among the strains with high level of $c f i A$ expression, only a minimum increase, or more often a decrease was observed between the $\operatorname{logRQ}$ values at $30 \mathrm{~min}$ and $60 \mathrm{~min}$, indicating that the full hydrolysis of imipenem was achieved already after $30 \mathrm{~min}$. Conversely, the strains with low level of cfiA expression showed a significant increase of $\operatorname{logRQ}$ between $30 \mathrm{~min}$ and $60 \mathrm{~min}$, suggesting that they required more time to hydrolyze completely the imipenem. No significant increase between the $\log R Q$ values at $30 \mathrm{~min}$ and $60 \mathrm{~min}$ was observed among cfiA-negative strains, proving, beyond the absence of any bacterial hydrolytic activity, a very good stability of imipenem molecules in the assay (Fig. 1, Table 3).

\subsection{Comparison of the results obtained with three different carbapenemase detection method}

The Carba NP test detected carbapenemase activity in 6 of 29 (20.7\%) Division II B. fragilis strains. It gave indeterminate result for 17 (58.6\%) strains, and was negative for 6 isolates most of them with meropenem MIC $\leq 2 \mathrm{mg} / \mathrm{L}$. Disk-diffusion synergy test detected the B. fragilis carbapenemase in 23 of 29 (79.3\%) cases with all the isolates which had a meropenem MIC $\geq 4 \mathrm{mg} / \mathrm{L}$ with one exception, strain BF95 (Table 4). Six strains (identical to the Carba NP negatives) gave no interpretable result by synergy test. The strains detected by both these methods corresponded to those characterized by higher meropenem MIC values ( $\geq 8 \mathrm{mg} / \mathrm{L}$ ) (Table 4 ). The capability of Carba NP and disk-diffusion synergy test to detect carbapenemase activity in $B$. fragilis strains belonging to Division II was dependent on the meropenem MICs, thus strains with lower MIC values were not detected, while STAR-Carba assay detected carbapenemase-activity in all strains, regardless their MICs.

\section{Discussion}

During this study, the largest number ever of $B$. fragilis clinical isolates was tested by MALDI-TOF MS for their status to belong to Division I/II. Typing was performed using the spectra recorded for routine identification. We confirmed previous data, underlining that MALDI-TOF MS-based subtyping immediately performed during species identification offers the possibility to give preliminary information to clinicians about the presence of carbapenemase production in $B$. fragilis $[31,32,39]$. Previous studies found that the prevalence of cfiA containing $B$. fragilis strains, belonging to Division II, is about $2-7 \%$ in the $B$. fragilis population obtained from clinical specimens [32,40-43], with only one study reporting a much higher prevalence (38\% in Turkey) [37]. Regarding prevalence among blood cultures, a recent study from Denmark shows reduced susceptibility to carbapenems in $10.2 \%$ of isolates from blood cultures [36]. In this study, interestingly we found higher percentage of Division II isolates among the strains tested in Bologna (10.1\%) compared to those, which were tested in Dortmund (7.6\%). An even bigger difference was found for strains isolated from positive blood cultures in the two cities $(15.2 \%$ in Bologna and 6\% in Dortmund, respectively). This may be due to the number and composition of the specimens received in the two different laboratories, but differences in usage of carbapenems in clinical practice may also contribute.

As viable isolates were only available from 2014 onwards, a subset of 70 isolates from both study sites, categorized by MALDITOF MS analysis to belong to Division II, was confirmed by PCR. Further 73 isolates classified as Division I by MALDI-TOF MS were randomly selected from both test sites for PCR analysis (Table 1). Similar to earlier studies [31,32], PCR confirmed the presence of the 


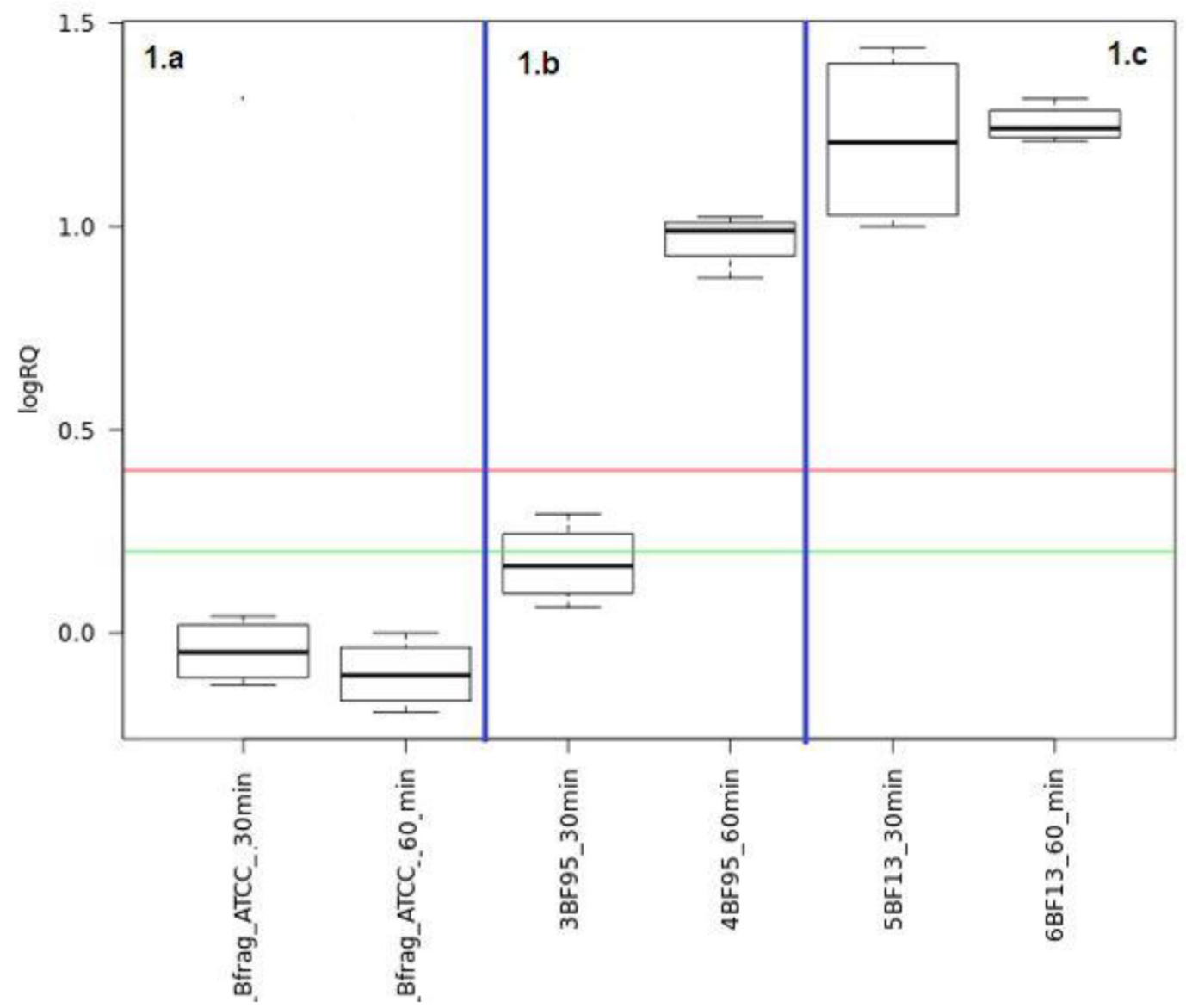

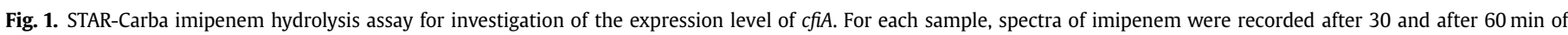
incubation with the bacterial strain tested.

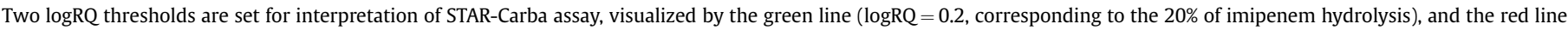

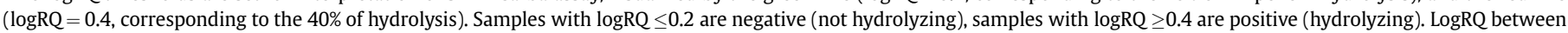
0.2 and 0.4 indicate an ambiguous degree of hydrolysis, that requires to repeat the test.

1a. Negative control: the sample was not hydrolyzing imipenem $(\log R Q<0.2)$ both after 30 and $60 \mathrm{~min}$.

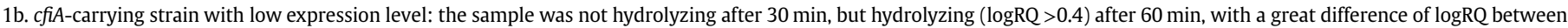
the two measurements.

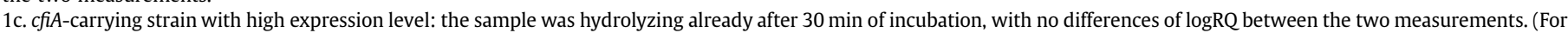
interpretation of the references to colour in this figure legend, the reader is referred to the Web version of this article.)

Table 3

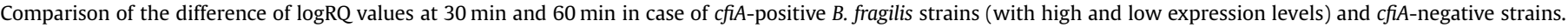

\begin{tabular}{|c|c|c|c|}
\hline \multirow[t]{2}{*}{ B. fragilis strains } & \multicolumn{3}{|l|}{ Average (range) } \\
\hline & $\operatorname{LogRQ}_{30 \min }$ & $\log _{60 \mathrm{~min}}$ & $\Delta \log R Q_{60 \min -30 \min }$ \\
\hline cfiA-positive - high expression ${ }^{\mathrm{a}}(\mathrm{n}=11)$ & $1.28(1.05 \rightarrow 1.44)$ & $1.14(0.97 \rightarrow 1.47)$ & $-0.14(-0.44 \rightarrow+0.04)$ \\
\hline cfiA-positive - low expression ${ }^{\mathrm{b}}(\mathrm{n}=59)$ & $0.43(-0.69 \rightarrow 1.38)$ & $1.11(0.48 \rightarrow 1.78)$ & $+0.58(-0.03 \rightarrow 1.57)$ \\
\hline cfiA-negative $(\mathrm{n}=33)$ & $-0.44(-0.74 \rightarrow-0.04)$ & $-0.41(-0.74 \rightarrow-0.11)$ & $+0.03(-0.33 \rightarrow+0.65)$ \\
\hline
\end{tabular}

a Meropenem MIC $\geq 16 \mathrm{mg} / \mathrm{L}$.

b Meropenem MIC $<16 \mathrm{mg} / \mathrm{L}$.

cfiA gene in all B. fragilis strains typed by MALDI-TOF MS to belong to Division II. No positive PCR result was found among the isolates categorized to belong to Division I (Table 1).

We also performed susceptibility testing for these PCR confirmed strains, and found for Division II (cfiA positive) isolates meropenem MICs between 0.125 and $\geq 32 \mathrm{mg} / \mathrm{L}$, while imipenem MICs were between 0.062 and $\geq 32 \mathrm{mg} / \mathrm{L}$ (Table 2). Comparing the two agents, in most cases the MIC values for meropenem were 1-4 dilution steps higher than the MIC values for imipenem. This also has been described in the literature [21] and is also in concordance with the suggestion by EUCAST to use meropenem for the determination of carbapenem resistance in B. fragilis [43].

Previous surveillance studies have found that highly resistant strains among clinical isolates of $B$. fragilis are rare (reports of $0.2-0.3 \%$ in Europe and USA [44] and ca. $1 \%$ in Japan [42]). In our subset of $B$. fragilis isolates, 11 of 70 Division II strains proved to be highly resistant, with meropenem and imipenem MICs $\geq 16 \mathrm{mg} / \mathrm{L}$, corresponding to a prevalence of $1.0 \%$ regarding Dortmund, but slightly higher (1.7\%) among all B. fragilis strains isolated in Bologna, suggesting, again, a different epidemiological/clinical context in the 
Table 4

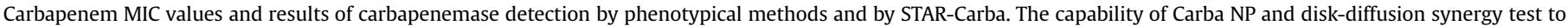

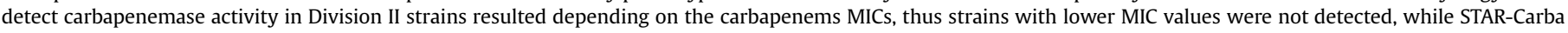
assay detected carbapenemase-activity in all strains, regardless their MICs.

\begin{tabular}{|c|c|c|c|c|c|}
\hline \multirow[t]{2}{*}{ Isolates $(\mathrm{n}=29)$} & \multicolumn{2}{|l|}{$\mathrm{MIC}(\mathrm{mg} / \mathrm{L})$} & \multicolumn{3}{|c|}{ Detection of carbapenemase activity by } \\
\hline & Meropenem MIC & Imipenem MIC & Carba NP Test & Disk-diffusion synergytest & STAR-Carba test \\
\hline BF-02 & $>32$ & $>32$ & POSITIVE & POSITIVE & POSITIVE HIGH EXPRESSION \\
\hline $\mathrm{BF}-13$ & $>32$ & $>32$ & POSITIVE & POSITIVE & POSITIVE HIGH EXPRESSION \\
\hline BF-22 & $>32$ & $>32$ & POSITIVE & POSITIVE & POSITIVE HIGH EXPRESSION \\
\hline BF-188 & $>32$ & $>32$ & POSITIVE & POSITIVE & POSITIVE HIGH EXPRESSION \\
\hline BF-04 & 16 & 4 & POSITIVE & POSITIVE & POSITIVE low expression \\
\hline BF-14 & 8 & 4 & indeterminate & POSITIVE & POSITIVE low expression \\
\hline $\mathrm{BF}-35$ & 8 & 8 & POSITIVE & POSITIVE & POSITIVE low expression \\
\hline BF-44 & 8 & 4 & indeterminate & POSITIVE & POSITIVE low expression \\
\hline BF-132 & 8 & 0.50 & indeterminate & POSITIVE & POSITIVE low expression \\
\hline $\mathrm{BF}-27$ & 4 & 0.12 & indeterminate & POSITIVE & POSITIVE low expression \\
\hline BF-63 & 4 & 0.12 & indeterminate & POSITIVE & POSITIVE lowexpression \\
\hline BF-95 & 4 & 0.12 & negative & not interpretable & POSITIVE lowexpression \\
\hline BF-158 & 4 & 4 & indeterminate & POSITIVE & POSITIVE low expression \\
\hline BF-169 & 4 & 0.12 & indeterminate & POSITIVE & POSITIVE low expression \\
\hline BF-178 & 4 & 0.12 & indeterminate & POSITIVE & POSITIVE low expression \\
\hline BF-194 & 4 & 4 & indeterminate & POSITIVE & POSITIVE low expression \\
\hline BF-199 & 4 & 4 & indeterminate & POSITIVE & POSITIVE low expression \\
\hline BF-231 & 4 & 4 & indeterminate & POSITIVE & POSITIVE low expression \\
\hline BF-236 & 4 & 0.12 & indeterminate & POSITIVE & POSITIVE low expression \\
\hline BF-237 & 4 & 0.25 & indeterminate & POSITIVE & POSITIVE low expression \\
\hline BF-279 & 4 & 4 & indeterminate & POSITIVE & POSITIVE low expression \\
\hline $\mathrm{BF}-313$ & 4 & 0.25 & indeterminate & POSITIVE & POSITIVE low expression \\
\hline BF-322 & 4 & 2 & indeterminate & POSITIVE & POSITIVE low expression \\
\hline $\mathrm{BF}-323$ & 4 & 0.50 & indeterminate & POSITIVE & POSITIVE low expression \\
\hline BF-60 & 2 & 0.12 & negative & not interpretable & POSITIVE low expression \\
\hline BF-240 & 2 & 4 & negative & not interpretable & POSITIVE low expression \\
\hline BF-275 & 2 & 0.12 & negative & not interpretable & POSITIVE low expression \\
\hline BF-277 & 2 & 0.12 & negative & not interpretable & POSITIVE low expression \\
\hline BF-205 & 1 & 0.50 & negative & not interpretable & POSITIVE low expression \\
\hline
\end{tabular}

two datasets. The further investigation for IS elements showed that only 7 of our 11 highly resistant Division II strains harboured such promoters upstream the cfiA resistance gene. This has been described earlier $[14,19,35]$ and underlines that high carbapenem resistance may also be due to other, yet unknown, cellular factors increasing the expression of $c f i A$.

Confirmation test using imipenem hydrolysis and MS measurements by the MBT STAR-Carba assay verified the carbapenemase activity in all cfiA-positive strains, with considerable differences between the $c$ fiA positive isolates with high or low MICs for carbapenems. This finding confirms that the cfiA-encoded enzyme is the dominating carbapenemase in $B$. fragilis, even if the gene is not fully expressed. These results are in concordance with the earlier results of ertapenem hydrolysis assay carried out with $B$. fragilis strains belonging to Division II with the cfiA gene published by Johansson et al. [33,34], but the method we investigated here is faster, thanks to the usage of imipenem instead of ertapenem. Moreover, the investigated STAR-Carba assay is available as a ready-to-use kit that allows the prompt confirmation of the carbapenemase-production in $B$. fragilis strains immediately after their classification as belonging to Division II by MALDI-TOF MS subtyping. This may be of great importance for clinical isolates involved in life-threatening infections. Further, the rate of imipenem hydrolysis assay enabled a separation of the strains into fasthydrolyzers (full hydrolysis of imipenem achieved after $30 \mathrm{~min}$ ) and slow hydrolyzers (full hydrolysis of imipenem achieved after $60 \mathrm{~min}$ ) as two distinct groups. With regard to the level of resistance, these two groups corresponded to the strains with high MICs (11 isolates), and with medium to low MICs (59 isolates), respectively. Hence, the possibility to characterize the Division II isolates in terms of cfiA-expression level by MALDI-TOF MS applications has been demonstrated for the first time in this study. The cfiA expression levels proved to be strongly related with the MIC interpretative categories (strains with high level of expression fell in the "resistant" category, while strains with low level of expression fell in the "intermediate" or "susceptible" category, according to EUCAST breakpoints). Hence, the use of this novel Biotyper application could directly affect the antibiotic treatment. In fact, according with the EUCAST definition of "intermediate", and for similarity with the EUCAST guidelines for other kind of carbapenemase-producing bacteria, carbapenem agents can still be administered for strains that result intermediate or susceptible (corresponding, in this study, to the ones which exhibit cfiA low expression level), but should be avoided for strains exhibiting high cfiA expression level (corresponding, in this study, only to the resistant ones).

We also compared the performance of the MS-based workflow for detection of carbapenemase activity with the workflow which is applied to detect carbapenemase-production in Enterobacteriaceae in Bologna, adapted for anaerobes. The MALDI-based workflow showed to be by far superior to the potential application of the methods currently available in this laboratory. Compared to conventional methods, the MALDI-TOF MS approach detected 7/29 cfiA-positive strains which were not found by disk diffusion synergy test, and 24/29 that were negative using Carba NP. Although strains with high and medium meropenem MICs were detected by synergy test after $24-48 \mathrm{~h}$, all the low MIC strains ( $\leq 2 \mathrm{mg} / \mathrm{l}$ ) would not have been detected. This might be of particular importance, because activation of cfiA expression under selective pressure during antibiotic treatment may happen $[20,38]$.

The novel full MALDI-TOF MS-based workflow to identify and type $B$. fragilis and even to detect the level of carbapenemase activity of the isolates was found to be more precise compared to the methods currently available for routine practice. Furthermore, the 
new approach was much faster for carbapenemase detection in B. fragilis. The whole approach takes about $2 \mathrm{~h}$ of time. Moreover, frequently subculturing is not necessary, since a few colonies are enough to perform both MALDI typing and imipenem hydrolysis assay. Further studies are needed to confirm the applicability of this concept of subtyping also to other bacteria and resistance mechanisms, any time that a resistance-specific peaks in the mass spectra are found.

\section{Acknowledgment}

This study was partly supported by an ESGAI grant for JS.

\section{References}

[1] H.M. Wexler, Bacteroides: the good, the bad, and the nitty-gritty, Clin. Microbiol. Rev. 20 (2007) 593-621.

[2] I. Brook, Spectrum and treatment of anaerobic infections, J. Infect. Chemother. 22 (2016) 1-13.

[3] D.W. Hecht, Prevalence of antibiotic resistance in anaerobic bacteria: worrisome developments, Clin. Infect. Dis. 39 (2004) 92-97.

[4] E. Nagy, E. Urbán, C.E. Nord, ESCMID study group on antimicrobial resistance in anaerobic bacteria, antimicrobial susceptibility of Bacteroides fragilis group isolates in Europe: 20 years of experience, Clin. Microbiol. Infect. 17 (2011) 371-379.

[5] I. Phillips, A. King, C.E. Nord, B. Hoffstedt, Antibiotic sensitivity of Bacteroides fragilis group in Europe, Eur. J. Clin. Microbiol. Infect. Dis. 11 (1992) 292-304.

[6] M. Hedberg, C.E. Nord, Antimicrobial susceptibility of Bacteroides fragilis group isolates in Europe, Clin. Microbiol. Infect. 9 (2003) 475-488.

[7] Centers for Disease Control and Prevention (CDC), Multidrug-resistant Bacteroides fragilis, Seattle, Washington, 2013, MMWR Morb. Mortal. Wkly. Rep. 62 (2013) 694-696.

[8] G.N. Hartmeyer, J. Sóki, E. Nagy, U.S. Justesen, Multidrug-resistant Bacteroides fragilis group on the rise in Europe? J. Med. Microbiol. 61 (2012) 1784-1788.

[9] J. Sóki, M. Hedberg, S. Patrick, B. Bálint, R. Herczeg, I. Nagy, D.W. Hecht, E. Nagy, E. Urbán, Emergence and evolution of an international cluster of MDR Bacteroides fragilis isolates, J. Antimicrob. Chemother. 71 (2016) 2441-2448.

[10] D.R. Snydman, N.V. Jacobus, L.A. McDermott, Y. Golan, E.J.C. Goldstein, L. Harrell, S. Jenkins, D. Newton, C. Pierson, J. Rosenblatt, R. Venezia, S.L. Gorbach, A.M. Queenan, D.W. Hecht, Update on resistance of Bacteroides fragilis group and related species with special attention to carbapenems 20062009, Anaerobe 17 (2011) 147-151.

[11] C.Y. Liu, Y.T. Huang, C.H. Liao, L.C. Yen, H.Y. Lin, P.R. Hsueh, Increasing trends in antimicrobial resistance among clinically important anaerobes and Bacteroides fragilis isolates causing nosocomial infections: emerging resistance to carbapenems, Antimicrob. Agents Chemother. 52 (2008) 3161-3168.

[12] S.P. Hawser, M. Hackel, D.J. Hoban, Antibiotic susceptibility profiles of European Bacteroides fragilis with reduced carbapenem susceptibility, J. Antimicrob. Chemother. 65 (2010) 803-804.

[13] T. Goto, K. Tanaka, C. Minh Tran, K. Watanabe, Complete sequence of pBFUK1, a carbapenemase-harboring mobilizable plasmid from Bacteroides fragilis, and distribution of pBFUK1-like plasmids among carbapenem-resistant $B$. fragilis clinical isolates, J. Antibiot. (Tokyo) 66 (2013) 239-242.

[14] R. Ruimy, I. Podglajen, J. Breuil, R. Christen, E. Collatz, A recent fixation of cfiA genes in a monophyletic cluster of Bacteroides fragilis is correlated with the presence of multiple insertion elements, J. Bacteriol. 178 (1996) 1914-1918.

[15] B.A. Rasmussen, K. Bush, F.P. Tally, Antimicrobial resistance in Bacteroides, Clin. Infect. Dis.16 Suppl 4 (1993) S390-S400.

[16] J.S. Thompson, M.H. Malamy, Sequencing the gene for an imipenem-cefoxitinhydrolyzing enzyme (CfiA) from Bacteroides fragilis TAL2480 reveals strong similarity between CfiA and Bacillus cereus beta-lactamase II, J. Bacteriol. 172 (1990) 2584-2593.

[17] Z. Eitel, J. Sóki, E. Urbán, E. Nagy, ESCMID Study Group on Anaerobic Infection, the prevalence of antibiotic resistance genes in Bacteroides fragilis group strains isolated in different European countries, Anaerobe 21 (2013) 43-49.

[18] J. Sóki, Extended role for insertion sequence elements in the antibiotic resistance of Bacteroides, World J. Clin. Infect. Dis. 3 (2013) 1-12.

[19] J. Sóki, R. Edwards, E. Urbán, E. Fodor, Z. Beer, E. Nagy, Screening of isolates from faeces for carbapenem-resistant Bacteroides strains; existence of strains with novel types of resistance mechanisms, Int. J. Antimicrob. Agents 24 (2004) 450-454.

[20] I. Podglajen, J. Breuil, E. Collatz, Insertion of a novel DNA sequence, IS1186, upstream of the silent carbapenemase gene cfiA, promotes expression of carbapenem resistance in clinical isolates of Bacteroides fragilis, Mol. Microbiol. 12 (1994) 105-114.

[21] J. Sóki, R. Edwards, M. Hedberg, H. Fang, E. Nagy, C.E. Nord, ESCMID study group on antimicrobial resistance in anaerobic bacteria, examination of cfiA- mediated carbapenem resistance in Bacteroides fragilis strains from a european antibiotic susceptibility survey, Int. J. Antimicrob. Agents 28 (2006) 497-502.

[22] S.M. Finegold, Perspective on susceptibility testing of anaerobic bacteria, Clin. Infect. Dis. 25 (Suppl 2) (1997) S251-S253.

[23] E.J.C. Goldstein, D.M. Citron, P.J. Goldman, R.J. Goldman, National hospital survey of anaerobic culture and susceptibility methods: III, Anaerobe 14 (2008) 68-72.

[24] CLSI - Clinical Laboratory Standards Institute, M11A8-Methods for Antimicrobial Susceptibility Testing of Anaerobic Bacteria, 2012. https://clsi.org/ standards/products/microbiology/documents/m11/. (Accessed 17 December 2017).

[25] P. Bogaerts, A. Engelhardt, C. Berhin, L. Bylund, P. Ho, A. Yusof, Y. Glupczynski, Evaluation of a new meropenem-EDTA double-ended Etest strip for the detection of the cfiAmetallo-beta-lactamase gene in clinical isolates of Bacteroides fragilis, Clin. Microbiol. Infect. 14 (2008) 973-977.

[26] A.S. Schwensen, Z. Acar, T.V. Sydenham, Ä.C. Johansson, U.S. Justesen, Phenotypic detection of the cfiA metallo- $\beta$-lactamase in Bacteroides fragilis with the meropenem-EDTA double-ended Etest and the ROSCO KPC/MBL Confirm Kit, J. Antimicrob. Chemother. 72 (2) (2017) 437-440.

[27] T.V. Sydenham, J. Sóki, H. Hasman, M. Wang, U.S. Justesen, ESGAI (ESCMID Study Group on Anaerobic Infections), Identification of antimicrobial resistance genes in multidrug-resistant clinical Bacteroides fragilis isolates by whole genome shotgun sequencing, Anaerobe 31 (2015) 59-64.

[28] N. Handal, S. Bakken Jørgensen, H. Smith Tunsjø, B.O. Johnsen, T.M. Leegaard, Anaerobic blood culture isolates in a Norwegian university hospital: identification by MALDI-TOF MS vs 16S rRNA sequencing and antimicrobial susceptibility profiles, APMIS Acta Pathol. Microbiol. Immunol. Scand 123 (2015) $749-758$.

[29] M. Kostrzewa, E. Nagy, How MALDI-TOF mass spectrometry can aid diagnosis of hard-to-identify pathogenic bacteria, Expert Rev. Mol. Diagn 16 (2016) $509-511$.

[30] B. Rodríguez-Sánchez, L. Alcalá, M. Marín, A. Ruiz, E. Alonso, E. Bouza, Evaluation of MALDI-TOF MS (Matrix-Assisted laser desorption-ionization timeof-flight mass spectrometry) for routine identification of anaerobic bacteria, Anaerobe 42 (2016) 101-107.

[31] E. Nagy, S. Becker, J. Sóki, E. Urbán, M. Kostrzewa, Differentiation of division 1 (cfiA-negative) and division II (cfiA-positive) Bacteroides fragilis strains by matrix-assisted laser desorption/ionization time-of-flight mass spectrometry, J. Med. Microbiol. 60 (2011) 1584-1590.

[32] I. Wybo, A. De Bel, O, Soetens, F. Echahidi, K. Vandoorslaer, M. Van Cauwenbergh, D. Piérard, Differentiation of cfiA-negative and cfiA-positive Bacteroides fragilis isolates by matrix-assisted laser desorption ionization-time of flight mass spectrometry, J. Clin. Microbiol. 49 (2011) 1961-1964.

[33] Å. Johansson, E. Nagy, J. Sóki, ESGAI (ESCMID Study Group on Anaerobic Infections), Detection of carbapenemase activities of Bacteroides fragilis strains with matrix-assisted laser desorption ionization-time of flight mass spectrometry (MALDI-TOF MS), Anaerobe 26 (2014) 49-52.

[34] Å. Johansson, E. Nagy, J. Sóki, Instant screening and verification of carbapenemase activity in Bacteroides fragilis in positive blood culture, using matrixassisted laser desorption ionization-time of flight mass spectrometry, J. Med. Microbiol. 63 (2014) 1105-1110.

[35] J. Sóki, E. Fodor, D.W. Echt, R. Edwards, V.O. Rotimi, I. Kerekes, E. Urbán, E. Nagy, Molecular characterization of imipenem-resistant, cfiA-positive Bacteroides fragilis isolates from the USA, Hungary and Kuwait, J. Med. Microbiol. 53 (2004) 413-419.

[36] U.S. Justesen, F. Hansen, C. Østergaard, H.C. Schønheyder, D.S. Hansen, L.E. Lemming, H. Schumacher, O. Heltberg, J.D. Knudsen, E. Dzajic, M. Arpi, A.M. Hammerum, High rates of reduced susceptibility in the Bacteroides fragilis group isolated from blood cultures - the first national survey in Denmark, Int. J. Antimicrob. Agents 42 (2013) 188-190.

[37] N.U. Toprak, O.D. Uzunkaya, J. Sóki, G. Soyletir, Susceptibility profiles and resistance genes for carbapenems (cfiA) and metronidazole (nim) among Bacteroides species in a Turkish University Hospital, Anaerobe 18 (2012) 169-171.

[38] I. Podglajen, J. Breuil, F. Bordon, L. Gutmann, E. Collatz, A silent carbapenemase gene in strains of Bacteroides fragilis can be expressed after a one-step mutation, FEMS Microbiol. Lett. 70 (1992) 21-29.

[39] P.L. Ho, C.Y. Yau, L.Y. Ho, J.H.K. Chen, E.L.Y. Lai, S.W.U. Lo, C.W.S. Tse, K.H. Chow, Rapid detection of cfiAmetallo- $\beta$-lactamase-producing Bacteroides fragilis by the combination of MALDI-TOF MS and CarbaNP, J. Clin. Pathol. 70 (2017) 868-873.

[40] S.A. Ferløv-Schwensen, T.V. Sydenham, K.C.M. Hansen, S.V. Hoegh, U.S. Justesen, Prevalence of antimicrobial resistance and the cfiA resistance gene in Danish Bacteroides fragilis group isolates since 1973, Int. J. Antimicrob. Agents 50 (2017) 552-556.

[41] I. Podglajen, J. Breuil, I. Casin, E. Collatz, Genotypic identification of two groups within the species Bacteroides fragilis by ribotyping and by analysis of PCRgenerated fragment patterns and insertion sequence content, J. Bacteriol. 177 (1995) 5270-5275.

[42] J. Sóki, E. Urban, I. Szöke, E. Fodor, E. Nagy, Prevalence of the carbapenemase gene (cfiA) among clinical and normal flora isolates of Bacteroides species in 
Hungary, J. Med. Microbiol. 49 (2000) 427-430.

[43] K. Yamazoe, N. Kato, H. Kato, K. Tanaka, Y. Katagiri, K. Watanabe, Distribution of the cfiA gene among Bacteroides fragilis strains in Japan and relatedness of cfiA to imipenem resistance, Antimicrob. Agents Chemother. 43 (1999) $2808-2810$.
[44] D.R. Snydman, N.V. Jacobus, L.A. McDermott, S. Supran, G.J. Cuchural S. Finegold, L. Harrell, D.W. Hecht, P. Iannini, S. Jenkins, C. Pierson, J. Rihs, S.L. Gorbach, Multicenter study of in vitro susceptibility of the Bacteroides fragilis group, 1995 to 1996, with comparison of resistance trends from 1990 to 1996, Antimicrob. Agents Chemother. 43 (1999) 2417-2422. 